\title{
Effects of Pb Treatment on Optical Properties of Aqueous CdSe
}

\section{Quantum Dots}

\author{
Cheng-Hsin Lu, ${ }^{1}$ Wan Y. Shih, ${ }^{2}$ and Wei-Heng Shih ${ }^{1 *}$ \\ ${ }^{1}$ Department of Materials Science and Engineering, Drexel University, Philadelphia, PA 19104, USA \\ ${ }^{2}$ School of Biomedical Engineering, Science and Health Systems, Drexel University, Philadelphia, PA 19104, USA \\ * Corresponding Author E-mail: shihwh@drexel.edu.
}

\section{Supporting Information}

The elemental analyses and optical responses of CdSe (4:3:1) QDs treated with different amount of $\mathrm{Pb}$ precursors, optical responses of purified and unpurified $\mathrm{CdSe}$ (4:3:1) QDs after Pb treatment, and the X-ray diffraction patterns of CdSe (4:1:1) versus $\mathrm{CdSe}(4: 1: 1) / 1 \mathrm{~Pb}$ were summarized as follows.

Table S1. The summary table of energy-dispersive X-ray spectroscopy (EDS) of untreated $\mathrm{CdSe}$ quantum dot powder versus $\mathrm{Pb}$-treated $\mathrm{CdSe}$ quantum dot powder. The molar ratios of QDs are MPA:Cd:Se $=(4: 3: 1)$ and $\mathrm{MPA}: \mathrm{Cd}: \mathrm{Se} / \mathrm{Pb}=(4: 3: 1 / 0.5),(4: 3: 1 / 1)$, $(4: 3: 1 / 1.5),(4: 3: 1 / 2)$, respectively. It can be seen that the $\mathrm{Pb} / \mathrm{Se}$ ratio results are basically consistent with the coating ratio showing the $\mathrm{Pb}$ ion are indeed added to the quantum dot surface.

\begin{tabular}{cccccc} 
& $\begin{array}{c}\text { CdSe } \\
(\mathbf{4 : 3 : 1 )}\end{array}$ & $\begin{array}{c}\text { CdSe/0.5Pb } \\
\mathbf{( 4 : 3 : 1 / 0 . 5 )}\end{array}$ & $\begin{array}{c}\mathbf{C d S e} / \mathbf{1 . 0 P b} \\
\mathbf{( 4 : 3 : 1 / 1 . 0 )}\end{array}$ & $\begin{array}{c}\text { CdSe/1.5Pb } \\
\mathbf{( 4 : 3 : 1 / 1 . 5 )}\end{array}$ & $\begin{array}{c}\mathbf{C d S e / 2 . 0 P b} \\
\mathbf{( 4 : 3 : 1 / 2 . 0 )}\end{array}$ \\
\hline Element & Attomic $\%$ & Atomic $\%$ & Atomic $\%$ & Atomic \% & Atomic \% \\
CdL & 38.41 & 36.53 & 30.61 & 27.40 & 26.00 \\
SeK & 14.10 & 14.03 & 10.83 & 8.65 & 9.10 \\
PbL & 0.15 & 7.67 & 10.79 & 12.47 & 17.78 \\
Others & 47.35 & 41.77 & 47.77 & 51.48 & 47.12 \\
Total & 100.0 & 100.0 & 100.0 & 100.0 & 100.0
\end{tabular}



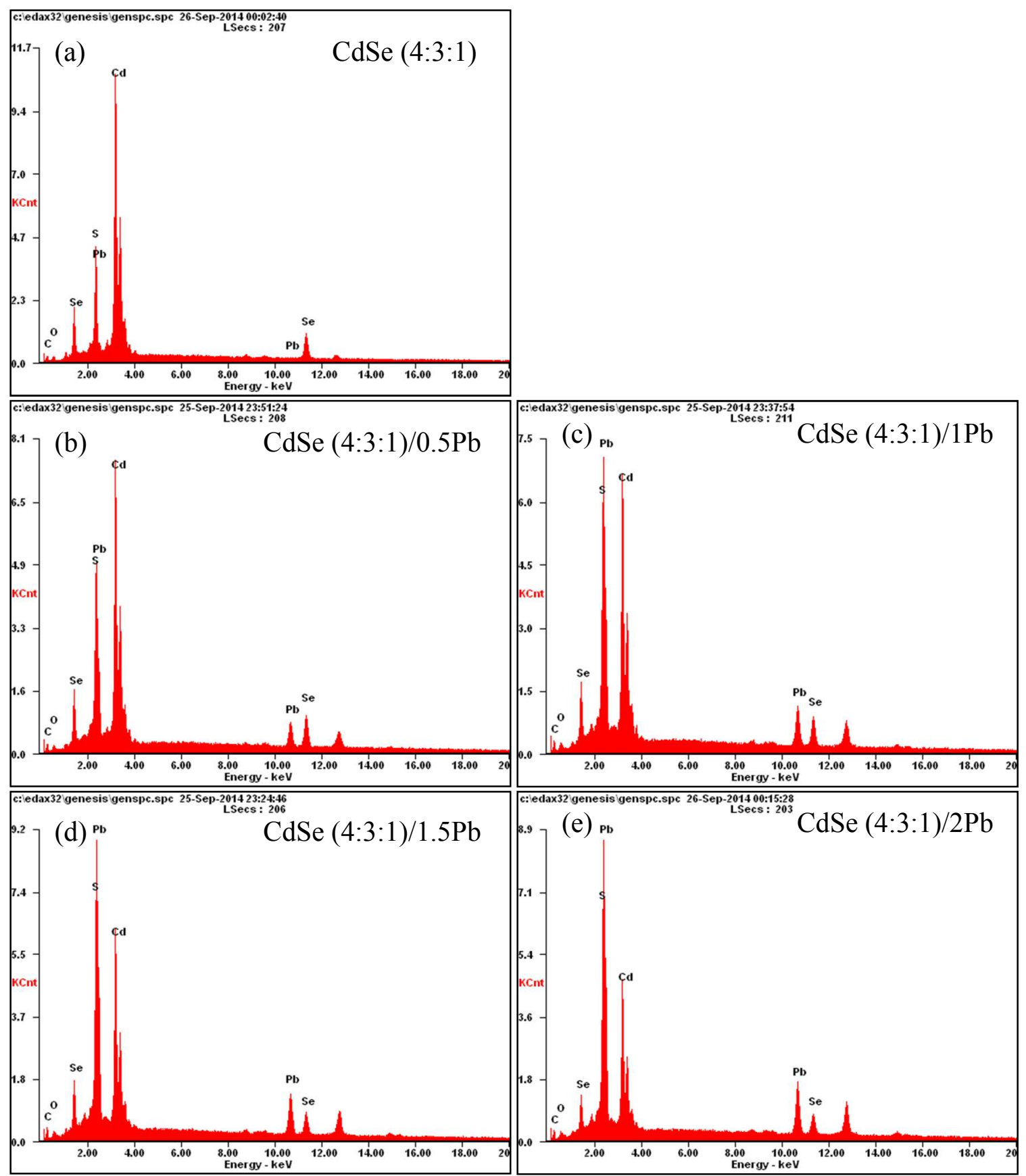

Figure S1. The energy-dispersive X-ray spectroscopy (EDS) of untreated CdSe quantum dot powder versus $\mathrm{Pb}$-treated $\mathrm{CdSe}$ quantum dot powder. The molar ratios of MPA:Cd:Se/Pb=(4:3:1/0) (a),(4:3:1/0.5) (b), (4:3:1/1) (c), (4:3:1/1.5) (d), and (4:3:1/2) (e), respectively. The sulfur signals "S" were contributed from the thiol group (R-SH) of MPA. Under high $\mathrm{pH}$ condition, the $\mathrm{H}$ of the thiol group will dissociate and result in $\mathrm{R}-\mathrm{S}^{-}$ ligand. The $\mathrm{R}-\mathrm{S}^{-}$will strongly bond to the excess $\mathrm{Cd}^{2+}$ surface and therefore the MPA won't be washed away after purification. 
(a)

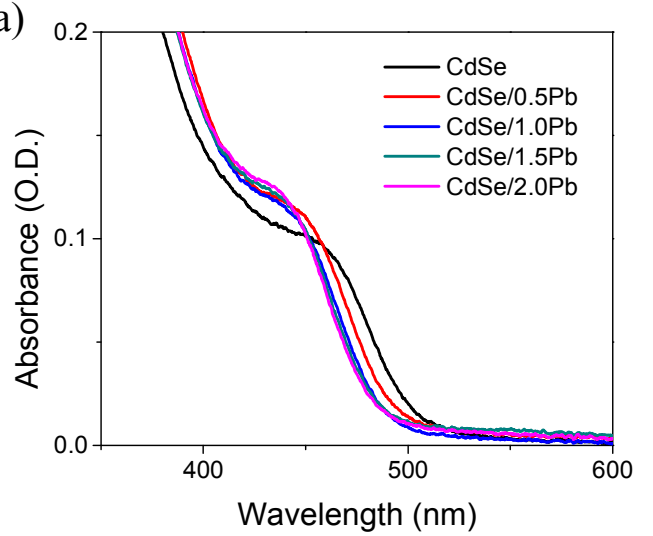

(b)

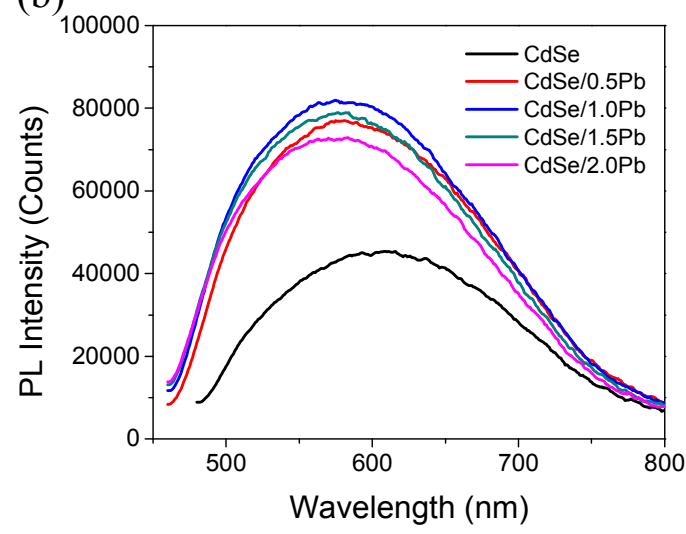

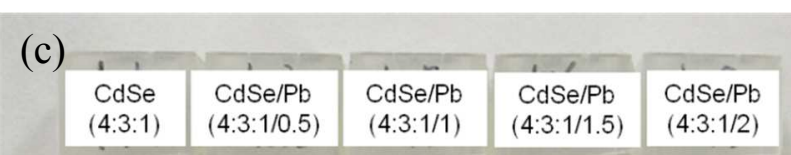
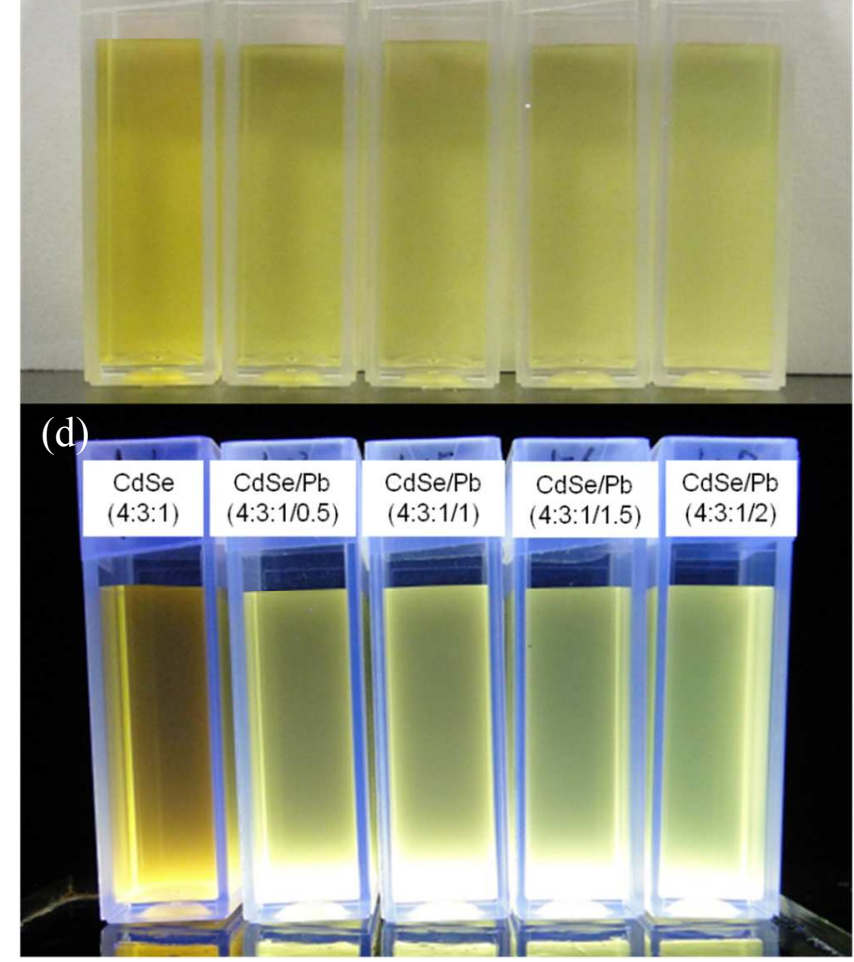

Figure S2. The (a) absorbance and (b) photoluminescence emission spectra of untreated CdSe quantum dot suspension with molar ratio of MPA:Cd:Se=(4:3:1) versus $\mathrm{Pb}$-treated CdSe QD with different amount of added $\mathrm{Pb}$ where molar ratio of $\mathrm{MPA}: \mathrm{Cd}: \mathrm{Se} / \mathrm{Pb}$ $=(4: 3: 1 / 0.5),(4: 3: 1 / 1),(4: 3: 1 / 1.5)$, and $(4: 3: 1 / 2)$. The appearances of CdSe and Pb-treated CdSe quantum dot suspensions under (c) ambient light and (d) ultraviolet lamp. 


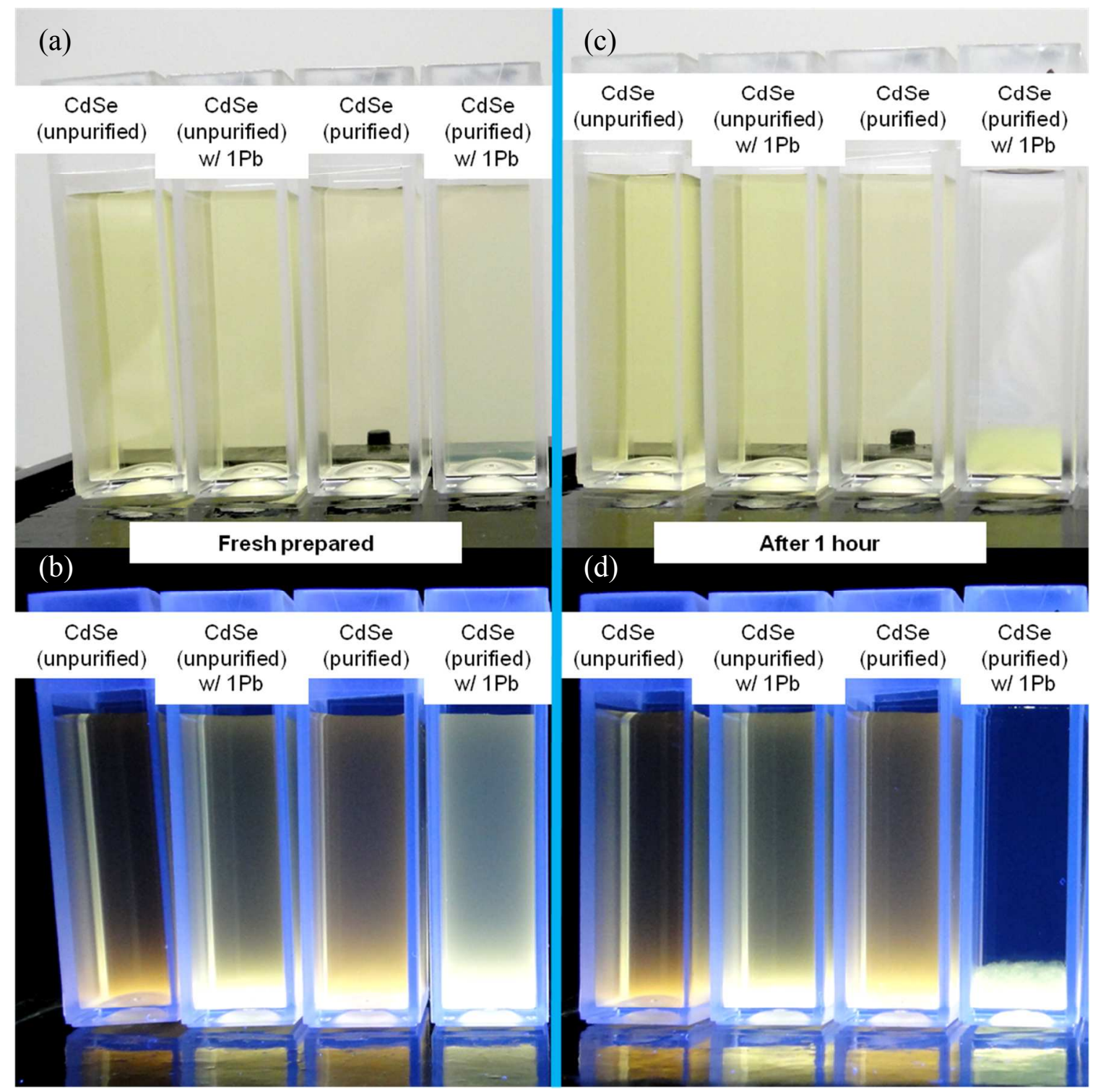

Figure S3. The appearance of unpurified and purified CdSe samples (with molar ratio of MPA:Cd:Se=4:3:1) before and after $\mathrm{Pb}$ treatment under ambient light (a)(c), and under ultraviolet lamp (b)(d). Pictures (a) and (b) show that the PL intensities of the freshly prepared $\mathrm{Pb}$-treated samples were enhanced for both purified and unpurified $\mathrm{CdSe}$ suspensions. Pictures (c) and (d) show that the purified CdSe suspension after treated with $\mathrm{Pb}$ is no longer stable and precipitated at the bottom after one hour due to the absence of excess MPA. 
(a) Unpurified CdSe vs. $\mathrm{CdSe} / 1 \mathrm{~Pb}$

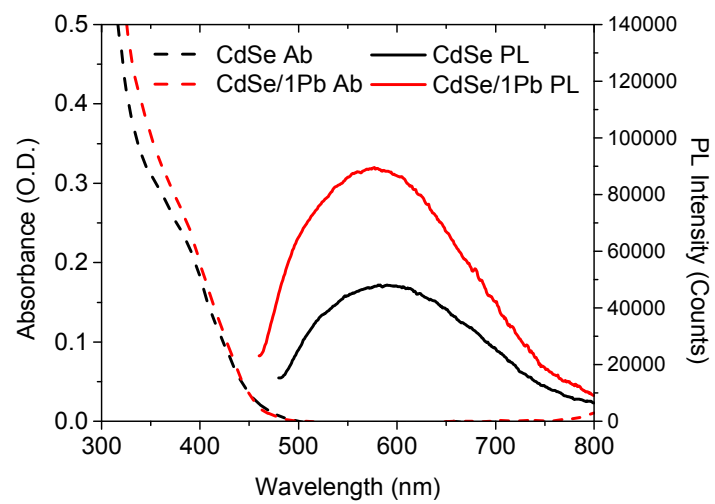

(b) Purified CdSe vs. CdSe/1Pb

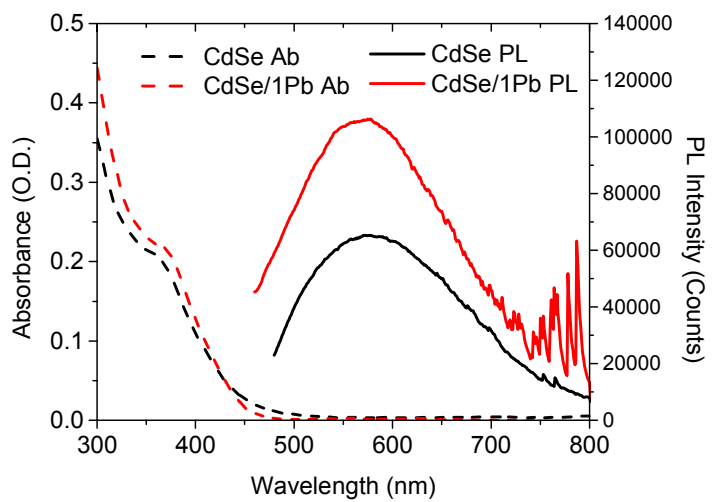

Figure S4. The absorbance spectra (dashed lines, left $Y$ axis) and photoluminescence emission spectra (solid lines, right $\mathrm{Y}$ axis) of freshly prepared (a) unpurified and (b) purified CdSe samples (with molar ratio of MPA:Cd:Se=4:3:1) before and after $\mathrm{Pb}$ treatment. Both unpurified and purified CdSe suspensions showed a similar PL enhancement behavior after $\mathrm{Pb}$ treatment. The noisy signal in the emission spectrum of $\mathrm{Pb}$-treated purified $\mathrm{CdSe}$ was probably due to the particle scattering from the aggregates due to the absence of excess MPA. 


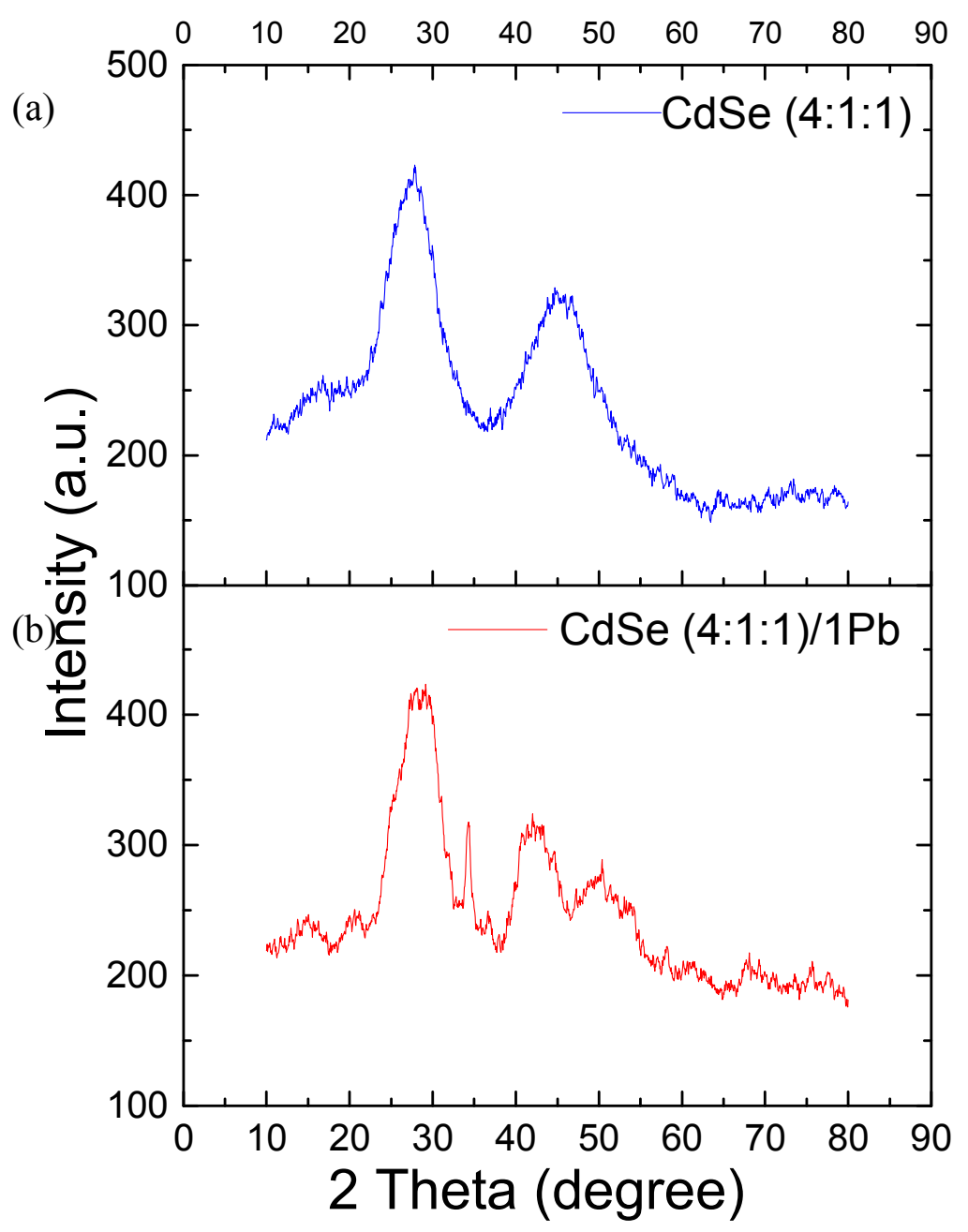

Figure S5. The X-ray diffraction (XRD) patterns of (a) untreated CdSe QDs (with molar ratio of MPA:Cd:Se=4:1:1), and (b) Pb-treated $\mathrm{CdSe}$ QDs (with molar ratio of MPA:Cd:Se/Pb =4:1:1/1). 